\title{
Damage Classification on Bridges using Backpropagation Neural Network
}

\author{
Victoria Ivy Tansil' ${ }^{1}$, Novita Hanafiah ${ }^{2}$, Alexander A. S. Gunawan ${ }^{3}$, \\ Dewi Suryani ${ }^{4 *}$ \\ ${ }^{1,2,3,4}$ Computer Science Department, School of Computer Science, \\ Bina Nusantara University, \\ Jakarta, Indonesia 11480 \\ victoria.tansil@binus.ac.id; novita.hanafiah@binus.ac.id; aagung@binus.edu; \\ dewi.suryani@binus.ac.id \\ *Correspondence: dewi.suryani@binus.ac.id
}

\begin{abstract}
Bridge structures can be damaged due to various factors such as pressure, vibration, temperature, etc. This study aims to detect damaged on bridges early so that accidents that can occur due to the damaged-on bridge can be avoided. The research method is divided into designing a model, building the model, and evaluating the model. The result of this research is a program that can classify healthy or damaged bridges using vibration data of tested points on bridges.
\end{abstract}

Keywords: structural health monitoring system; bridge structure; backpropagation neural network.

\section{INTRODUCTION}

Bridges are defined as building structures that connect routes or paths that are disconnected by the rivers, lakes, straits, canals, roads, or other crossings (Amir, 2013). Naturally, bridge construction resistance tends to decrease over time. Regular monitoring is needed to prevent a sudden collapse of bridges that can cause material and non-material harm. The collapse of bridges can disrupt the course of socio-economic activities in communities in various sectors such as education, commerce, and health.

To prevent the occurrence of collapsing bridges, it is necessary to monitor the health of the bridge or commonly known as Structural Health Monitoring System (SHMS). SHMS is the process of implementing a damage identification strategy for aerospace, civil, and mechanical infrastructure (Bagavathiappan et al, 2013). SHMS uses smart sensor technology to assist in identifying structural abnormalities by using measurable structural parameter responses. With careful planning in sensor placement, the
SHMS can collect the necessary data on the condition of the bridge. Such data as load rate, deformation rate, deflection, temperature, and other structural properties are changing with time (Hanus \& Harris, 2013). With this technology, bridge damage can be detected earlier so that repairs can be done with minimum cost and extend the life of the bridge.

To be able to know the bridge that needs to get monitoring or immediate handling, it is necessary to classify the damage. Classification will be done using the method of Artificial Neural Network (ANN). ANN is a model of intelligence inspired by the structure of the human brain and then implemented using a computer program capable of completing several calculation processes during the learning process (Rayata et al, 2014). ANN is used to classify certain patterns that are often known as linear separations. Hopefully, with this research, bridge damage can be analyzed quickly and precisely.

In this study, the main reference used there are two, namely (Gunawan, 2017) and (Suryanita \& Adnan, 2014). In previous research, the accuracy rate in predicting damage to a bridge structure only reached $84 \%$. Research on the first reference is done using the Support Vector Machine method, while research on the second reference is done by using the backpropagation method, with activation function using Sigmoid. All experiments will be conducted using the backpropagation method with the Sigmoid activation function and the Rectified Linear Units (ReLU) activation function.

Based on the background that has been mentioned above, shows the main purpose of this study is to produce a form of backpropagation neural network architecture to be used in predicting damage to the bridge structure with the best performance in this case. 
The rest of this paper is structured as follows. In Section II, we describe the methodology that is used in this study. Then, the details of all conducted experiments and the results are presented in Section III. Lastly, Section IV concludes our works in this paper and discusses the future work.

\section{METHODS}

The details of our methodology are described as follows.

\subsection{Data Collection Method}

Data is generated from numerical analysis of a sevendegree system of freedom (7-DOF), similar to that of Farrar and Worden (Farrar \& Worden, 2012). The system consists of seven masses weighing $1 \mathrm{~kg}$ each and eight springs with a stiffness of $1 \mathrm{~N} / \mathrm{m}$ each. Both ends of the system are fixed. The dynamic force, $f(t)$, has a random value placed on the center mass, . The force value $f(t)$ is taken from a normal probabilistic distribution with a mean of zero and a standard deviation of 0.09 . Initially, the random data $f(t)$ has a frequency of $25 \mathrm{~Hz}$, then filtered by using a Butterworth filter with a cutting frequency at $20 \mathrm{~Hz}$ and order 12 .

Damage to the structure is assumed to occur at the spring between $m_{3}$ and $m_{4}$ and only decreases the constant value of the spring. This numerical analysis is done by using a finite element application, that is LS-Dyna. The result of the analysis is the data of the displacement of the seven masses. Data is a sample of a constant increase every 0.1 seconds and with a duration of 360 seconds. For each structural condition, the analysis is repeated 500 times by making changes to the dynamic force $f(t)$.

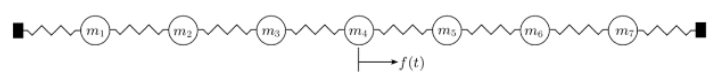

Figure 1. Seven Degree System of Freedom Model

The displacement data is denoted by $x(t)$. The value of $x(t)$ in the partition becomes a sub-signal $M$ of the same length $L$. Then, the sub- signal in the time domain form is converted into the frequency domain by using a discrete Fourier transform.

$$
X\left(f_{k}\right)=\sum_{i=0}^{N-1} x_{i} \cdot \exp \left(-j i 2 \pi f t_{s}\right)
$$

where $f \in\left[0, f_{s} / 2\right]$ and $f_{s}=1 / t_{s}$, called sample frequency and is discrete frequency of $f_{i}=i \cdot f_{s} / N$. To shorten expression, we use the symbol $X_{i}$ to denote $X\left(f_{i}\right)$.

After that, it through a process is called Power Spectral Density (PSD), in which the magnitude values obtained from the discrete Fourier transform are squared. Then averaging the value of Power Spectral Density (PSD). This whole process is a process of the Bartlett method.

$$
S_{i}(f)=\frac{1}{L M} \sum_{m=0}^{M-1}\left|X_{i}^{(m)}\right|^{2}
$$

where the signal length $N$ and the number of subsignals $M$ is related by $N=L M$, where $L$ is the length of the sub-signal.

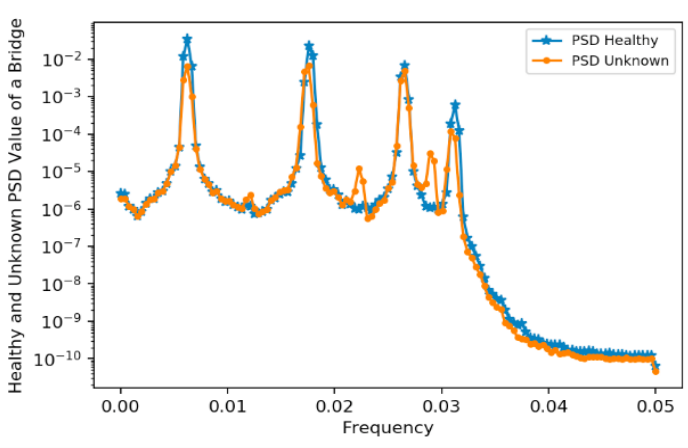

Figure 2. Example Result Graph of Bartlett Method

$F$ test along with a simple classification method has been used to perform damage detection. This method has been presented in theory (Fassois \& Sakellariou, 2009) and has been verified by experimentation (Kopsaftopoulos $\&$ Fassois, 2010). This method depends on the structure response that can be obtained by doing some point measurement. This method has three steps:

1. Statement of the null and alternative hypothesis for this case, namely:

$$
\begin{aligned}
& \mathrm{H}_{0}: S_{h}(w)=S_{u}(w) \\
& \mathrm{H}_{\mathrm{a}}: S_{h}(w) \neq S_{u}(w)
\end{aligned}
$$

where $S(w)$ symbol denotes the PSD. The subscript $h$ denotes the healthy condition. The subscript $u$ denotes the unknown-to-be-sought condition. The structure is assumed to be healthy if the $S_{u}$ value is equal or close to $S_{h}$ value, whereas it is considered to be damaged if the PSD has changed significantly.

2. $F$ test is done by using the value of PSD that has been obtained. $F$ test performs a comparison between $S_{h}(w)$ value dan $S_{u}(w)$ value. The calculation using the formula:

$$
F=\frac{\hat{s}_{h}(w) / S_{h}(w)}{\hat{s}_{u}(w) / S_{u}(w)}
$$

the denotes the estimated PSD. Under condition (3), equation (5) can be simplified to:

$$
F=\frac{\hat{s}_{h}(w)}{\hat{s}_{u}(w)}
$$

the structure is considered healthy when the $F$ test value approaches 1 . When the structure is damaged, the $F$ test value can deviate from 1 to very large or very small. The rate of change in the PSD value determines the value of the $F$ test.

3. Determine the upper and lower limits of the $F$ test value where the change in PSD can be categorized as significant or not. $F$ test results are very sensitive to disturbance and produce high fluctuations, often over the upper and lower limits on healthy structural conditions.

\subsection{Neural Network Method} follows:

This research is done by doing some stages as

\section{- Designing the model}

The first step is to design the model of the backpropagation neural network. At this stage determined 
many layers and nodes are used, the determination of learning rate, the activation function used, and the separation of training and testing dataset.

In this research, the method used is backpropagation. The neural network structure of backpropagation used in this research is 20 input nodes, $n$ hidden nodes, and 2 output nodes. In this study, the number of hidden nodes to be used include 5, 10, 20, and 50. The input node is the data taken from the simulation of vibration to the bridge. The output node is the data of $[1,0]$ and $[0,1]$, where $[1,0]$ represents undamaged bridges and $[0,1]$ represents the damaged bridge. The structure of the backpropagation can be seen in Figure 3.

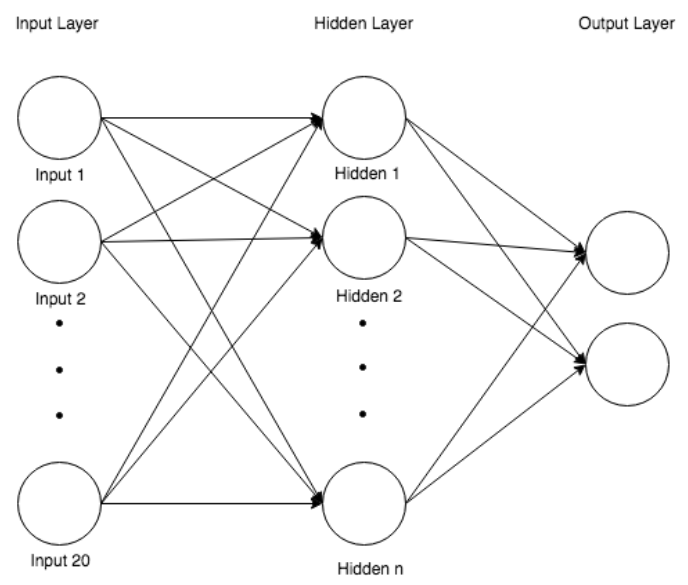

Figure 3. Backpropagation Architecture

\section{- Building the model}

After the model has been designed, then we begin to train the model using the training dataset based on specified architecture. Then, the next step is to test the built model using the test dataset.

\section{- $\quad$ Evaluating the model}

This step is to evaluate the performance of the model that has been created. If the results have not reached the optimal conditions in which the model can be used in case, not underfitting (much data from unattended training data) or overfitting (the model corresponds to almost all training data, but not in general terms), then we update the hyperparameter to increase its result.

\section{RESULTS AND DISCUSSION}

This research divided the experiments into several parts that are discussed in detail here.

\subsection{Bridge Analysis using Statistic}

$F$ statistics is a method that has been used to determine the extent of bridge damage. To determine whether a bridge is damaged or not, it is seen from the highest $F$ statistic and the lowest $F$ statistic. If the highest $F$ statistic value or the lowest $F$ statistic value exceeds the upper or lower limit of $F$ statistics, then the bridge is considered to be defective. Conversely, if the highest statistical $F$ value or the lowest $F$ statistic value is within the upper bound and lower bound, then the bridge is considered healthy.
As shown in Figures 4 and 5, the upper and lower borders are marked with a red line. The upper limit is 10 and the lower limit is 0.1 . In Figure 4, the structure is considered to be damaged because the $F$ value of the largest statistic passes the upper limit value, i.e., greater than 10 and the $F$ value of the smallest statistic passes the lower limit, i.e., less than 0.1 .

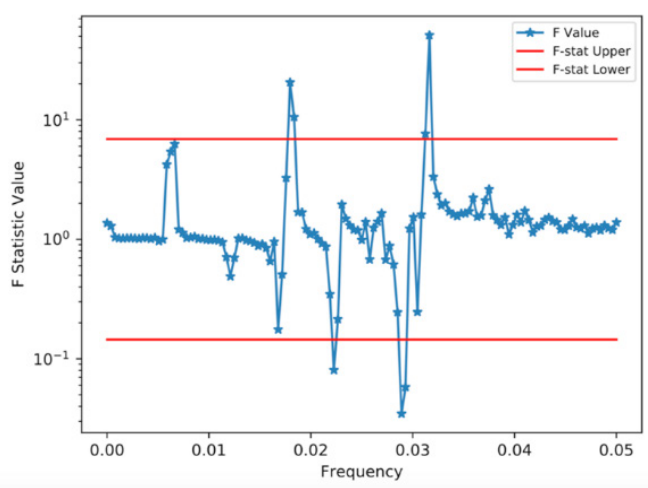

Figure 4. F Statistic Result for Damaged Bridge

On the other hand, Figure 5 depicts the healthy bridge structure due to the $F$ values of the largest and smallest statistics are within the lower and upper range.

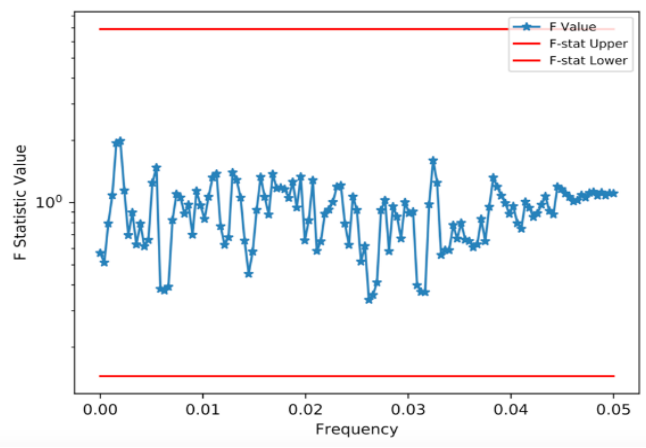

Figure 5. F Statistic Result for Healthy Bridge

Based on the research that has been done previously by (Gunawan, 2017), the level of accuracy by using statistic to bridge damage only reaches $72.6 \%$. The main reason is that this method often considers a healthy bridge structure as a broken bridge.

\subsection{Experimental Settings}

All experiments were performed using 3 layers, which consist of 1 input layer, 1 output layer, and 1 hidden layer. This experiment uses the backpropagation method. For each epoch, training is done 5 times to avoid biased results due to random data factors. The data sharing of training and testing used in this research is from a total of 17,493, that's means there are data for the training set and the remaining are for the test dataset. Accuracy is taken every multiple of 50 at the epochs value.

\subsection{Experimental Results}

In the first experiment, there were 4 experiments conducted, where experiments using the Sigmoid activation function on the hidden layer and the number of hidden nodes vary as shown in Table 1 . The initial weight parameters used a normal distribution with a mean value average of 0 , 
a standard deviation of 1 , and the initial bias value is zero. Training in the first experiment was performed using the following parameters:

Table 1. Default Parameter for $1^{\text {st }}$ Experiment

\begin{tabular}{lc}
\hline \multicolumn{1}{c}{ Parameter } & Value \\
\hline Epochs & 700.000 \\
\hline Learning Rate & $5 \times 10^{-3}$ \\
\hline Batch Size & 5.000 \\
\hline $\begin{array}{l}\text { Activation Function of } \\
\text { Output Layer }\end{array}$ & Softmax \\
\hline $\begin{array}{l}\text { Activation Function of } \\
\text { Hidden Layer }\end{array}$ & Sigmoid \\
\hline
\end{tabular}

Table 2. Changing Parameter for $1^{\text {st }}$ Experiment

\begin{tabular}{cc}
\hline Experiment & $\begin{array}{c}\text { Number of Hidden } \\
\text { Nodes }\end{array}$ \\
\hline I & 5 \\
\hline II & 10 \\
\hline III & 20 \\
\hline IV & 50 \\
\hline
\end{tabular}

In the second experiment, the activation function of the hidden layer is replaced by using Rectified Linear Units (ReLU). Similar to the first experiment, the weight values are initiated using a normal distribution with an average value of 0 and a standard deviation of 1 , the initial bias value is zero, and 4 experiments are performed by differentiating the number of hidden nodes. The hyperparameters are used in this second experiment as follows:

\begin{tabular}{lc} 
Table 3. Default Parameter for $2^{\text {nd }}$ Experimen \\
\cline { 2 - 2 } \multicolumn{1}{c}{ Parameter } & Value \\
\hline Epochs & 700.000 \\
\hline Learning Rate & $5 \times 10^{-3}$ \\
\hline Batch Size & 5.000 \\
\hline $\begin{array}{l}\text { Activation Function of } \\
\text { Output Layer }\end{array}$ & Softmax \\
\hline $\begin{array}{l}\text { Activation Function of } \\
\text { Hidden Layer }\end{array}$ & ReLu \\
\hline
\end{tabular}

Table 4. Changing Parameter for $2^{\text {nd }}$ Experiment

\begin{tabular}{cc}
\hline Experiment & $\begin{array}{c}\text { Number of Hidden } \\
\text { Nodes }\end{array}$ \\
\hline I & 5 \\
\hline II & 10 \\
\hline III & 20 \\
\hline IV & 50 \\
\hline
\end{tabular}

Accuracy value is obtained from the percentage between predicted output and actual output. In the first experiment can be seen in Figure 6, the converging value of epochs 8,000. In Figure 7, the converging value of epochs 8,000. In Figure 8, the value converges from 4,000, but on the 12,000 epochs, there is an increase. In Figure 9 the convergent value of 10,000 epochs. From these four experiments the best between first experiment accuracy I
(Figure 6) and first experiment accuracy II (Figure 7).

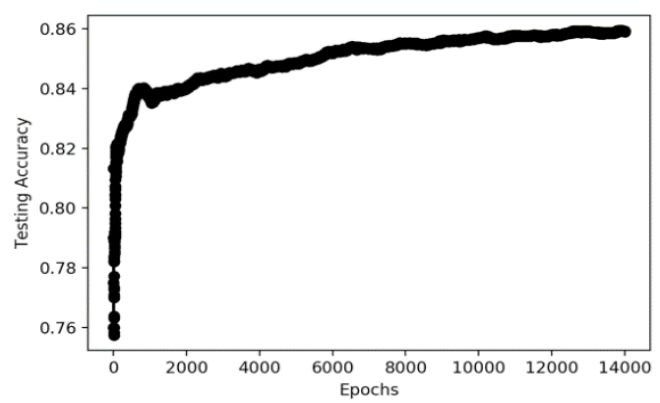

Figure 6. First Experiment Accuracy I

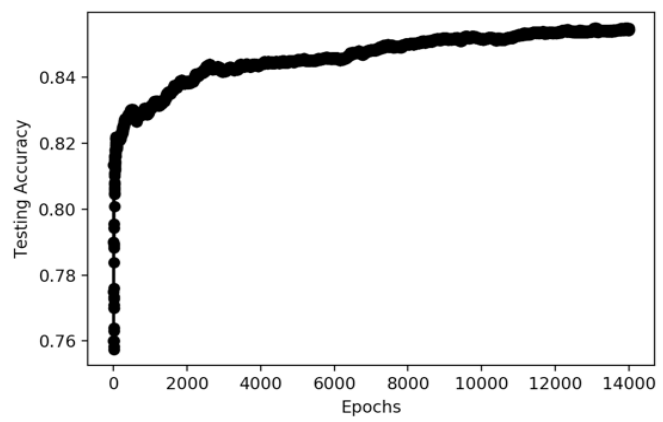

Figure 7. First Experiment Accuracy II

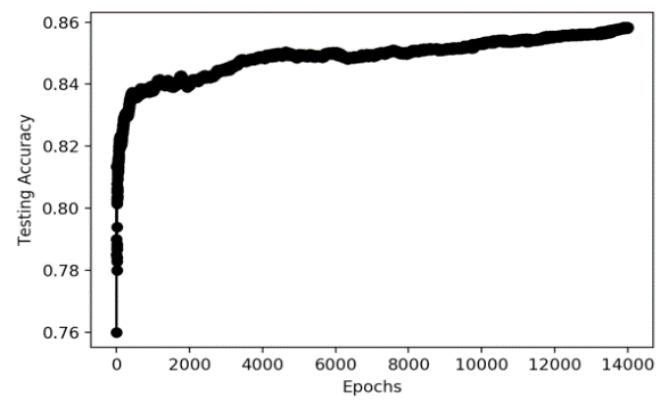

Figure 8. First Experiment Accuracy III

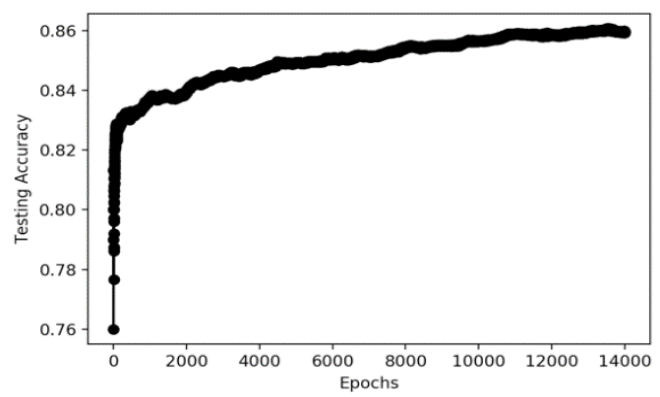

Figure 9. First Experiment Accuracy IV

In the second experiment can be seen in Figure 10, the converging value of epochs 4,000. In Figure 11, the convergent values of 4,000 epochs, however, are more unstable than the prior experiment (Figure 10). In Figure 12, the convergent value of 8,000 . In Figure 13 the convergent value of the 2,000 epochs, but at 10,000 epochs the accuracy falls from 0.87 to 0.2 . Therefore, the best of these four experiments is second experiment accuracy I (Figure 10). Then, when compared to the first experiment, the second 
experiment is much better because the convergent value is faster.

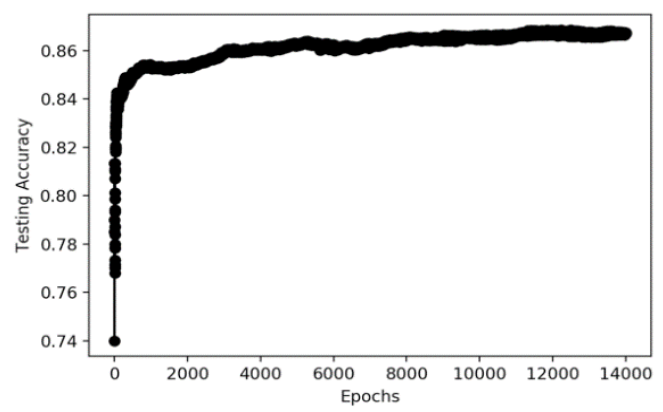

Figure 10. Second Experiment Accuracy I

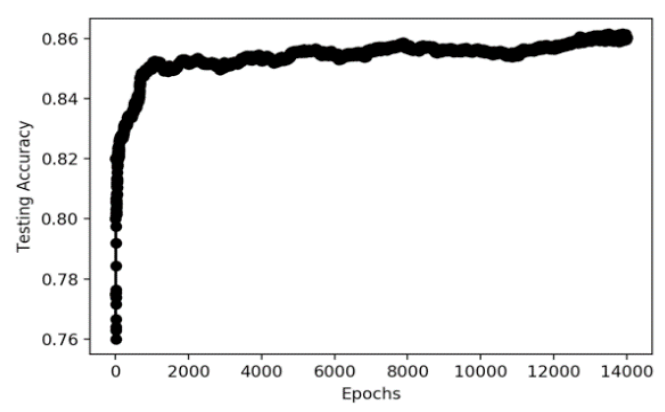

Figure 11. Second Experiment Accuracy II

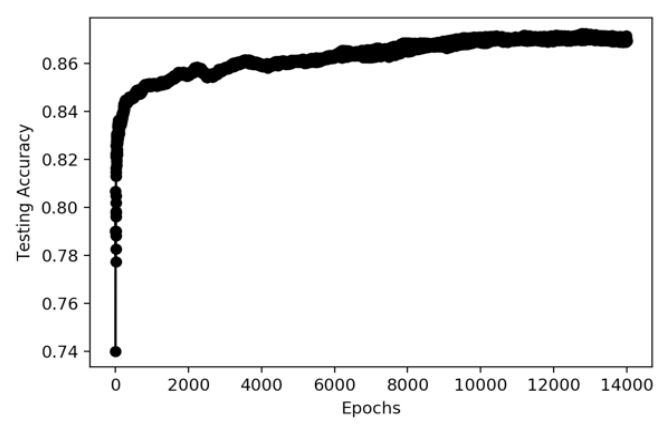

Figure 12. Second Experiment Accuracy III

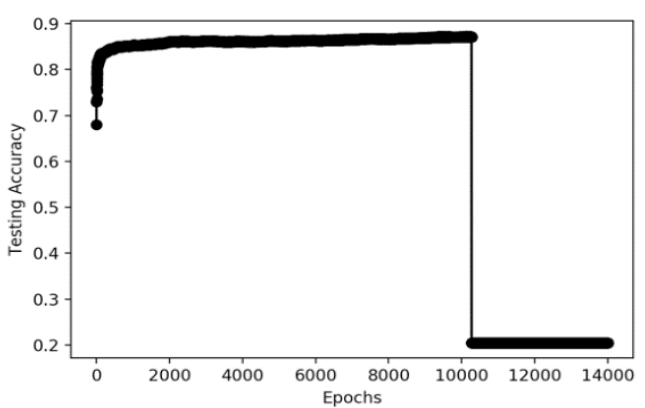

Figure 13. Second Experiment Accuracy IV

In the first experiment, the accuracy was between 0.8525 and 0.8559 . In the second experiment, the accuracy was between 0.8616 and 0.8767 with the most recent experimental accuracy of only 0.1973 . Then from the second experiment itself can be seen that the highest accuracy value is experiment III with an accuracy of 0.8767 or $87.67 \%$. Based on the average value of accuracy (Figure 14 ), it can be concluded that the second experiment where the activation function on the hidden layer using Rectified Linear Units (ReLU) has a higher accuracy value than the first experiment where the activation function on the hidden layer using Sigmoid.

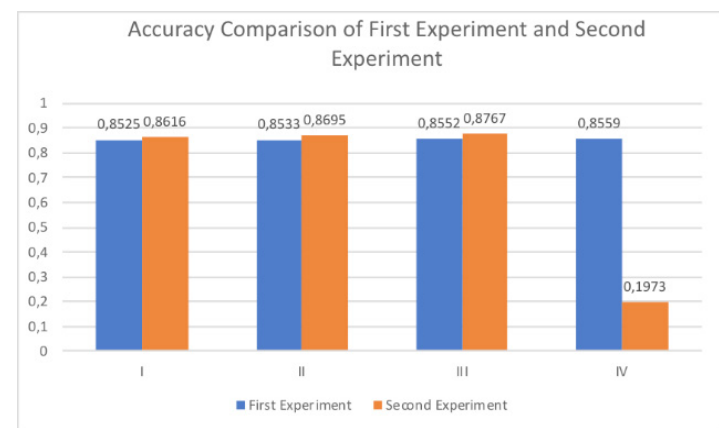

Figure 14. Accuracy Comparison of First Experiment and Second Experiment

\section{CONCLUSION}

Based on the results of research and analysis that have been done, then we conclude that ANN by using the backpropagation architecture can be used to predict the damage or not of a bridge structure. Moreover, the accuracy of our architecture has reached satisfactory results, i.e., $86 \%$. For the activation function, the ReLU activation function works better than the Sigmoid function in this case.

For the next development, we recommend classifying the damage of a bridge based on its level of damage and testing on the unknown data at once using one healthy data.

\section{REFERENCES}

Amir, A. (2013). Studi Keandalan Struktur Jembatan Sungai Tello (Lama) Berdasarkan Beban Lalu Lintas Umum dan Trailer Super Berat dengan Metode Moving Load. Makassar: Universitas Hasanuddin.

Bagavathiappan, S., Lahiri, B. B., Saravanan, T., Philip, J., \& Jayakumar, T. (2013). Infrared thermography for condition monitoring-a review. Infrared Physics \& Technology, 60, 35-55.

Farrar, C. R., \& Worden, K. (2012). Structural health monitoring: a machine learning perspective. John Wiley and Sons.

Fassois, S. D., \& Sakellariou, J. S. (2009). Encyclopedia of Structural Health Monitoring, Chapter 23: Statistical Time Series Methods for SHM. John Wiley and Sons.

Gunawan, F. E. (2017). Improving the Prediction Reliability of F-stat Method by using Linear Support Vector Machine for Structural Health Monitoring. Jakarta: Bina Nusantara University.

Hanus, M. J., \& Harris, A. T. (2013). Nanotechnology innovations for the construction industry. Progress in materials science, 58(7), 1056-1102. 
Kopsaftopoulos, F. P., \& Fassois, S. D. (2010). Vibration based health monitoring for a lightweight truss structure: experimental assessment of several statistical time series methods. Mechanical Systems and Signal Processing, 24(7), 1977-1997.

Rayata, F., Marveni, V. M., Putri, M., \& Sarfriadi, R. S. (2014). Artificial Neural Network. Padang: Universitas Negeri Padang.

Suryanita, R., \& Adnan, A. (2014). Early-warning system in bridge monitoring based on acceleration and displacement data domain. Transactions on Engineering Technologies, 157-169. 